\title{
TV/Series
}

$10 \mid 2016$

Guerres en séries (II)

\section{Les séries et la guerre (pistes de lectures et de réflexion)}

\section{Marjolaine Boutet}

\section{(2) OpenEdition \\ Journals}

Electronic version

URL: http://journals.openedition.org/tvseries/1946

DOI: 10.4000/tvseries.1946

ISSN: 2266-0909

\section{Publisher}

GRIC - Groupe de recherche Identités et Cultures

\section{Electronic reference}

Marjolaine Boutet, "Les séries et la guerre (pistes de lectures et de réflexion) », TV/Series [Online], 10 | 2016, Online since 05 December 2016, connection on 23 September 2020. URL : http:// journals.openedition.org/tvseries/1946; DOI : https://doi.org/10.4000/tvseries.1946

This text was automatically generated on 23 September 2020

\section{(c) (i) (3) $\Theta$}

$T V / S e r i e s$ est mis à disposition selon les termes de la licence Creative Commons Attribution - Pas d'Utilisation Commerciale - Pas de Modification 4.0 International 


\title{
Les séries et la guerre (pistes de lectures et de réflexion)
}

\author{
Marjolaine Boutet
}

\section{Guerre, mémoire, identité}

1 Avec les séries policières, les séries historiques sont le genre le plus populaire sur les télévisions du monde entier. Parce que la télévision s'est construite d'abord comme un média national et, particulièrement en Occident, comme un média de reconstruction nationale après la Seconde Guerre mondiale, elle fut aussi, plus ou moins consciemment, un vecteur important du " récit national ${ }^{1}$ ». Au sein de ces récits, les guerres occupent une place particulière, puisque, comme le veut l'adage, «l'Histoire appartient aux vainqueurs. » Le récit de guerre pourrait dès lors être interprété comme un moyen, ou une tentative, de s'approprier l'Histoire, de lui donner un/du sens.

Qu'il s'agisse de guerre contre un autre État ou de guerre civile, l'issue du conflit armé a toujours comme enjeu et comme résultat de redéfinir l'identité de la nation, de renforcer ou de remettre en cause l'image qu'elle a d'elle-même. Or cette image n'est pas fixe: le travail de mémoire porte notamment sur ces épisodes paroxystiques et, décennies après décennies, siècle après siècle, les interroge et les transforme ${ }^{2}$. « Nul ne consulte une archive sans projet d'explication, sans hypothèse de compréhension; et nul ne s'emploie à expliquer un cours d'événements sans recourir à une mise en forme littéraire expresse de caractère narratif, rhétorique ou imaginatif ${ }^{3}$ ", écrit Paul Ricœur dans La mémoire, l'histoire, l'oubli. Les rapports entre Histoire, récit, fiction et mémoire sont étroits et complexes, et le «mythe ", au sens de Roland Barthes, n'est jamais loin. C'est d'autant plus vrai dans le cas des récits populaires et répétitifs que sont les séries télévisées, qui produisent aussi, par leur dimension audiovisuelle, un puissant « effet de réel $»^{4}$.

Dès lors, les séries télévisées apparaissent comme des objets importants d'expression, de négociation, voire de fixation de la mémoire. L'évolution récente vers le rejet du manichéisme et la construction de narrations de plus en plus complexes, multipliant les 
focales et les points de vue, maniant l'ellipse et les ruptures de rythme, permet à nombre de séries produites au XXI ${ }^{\mathrm{e}}$ siècle de proposer un discours plus élaboré sur le passé, voire de se présenter explicitement comme un simple discours sur une période révolue dont il ne nous reste de toute façon que des traces ${ }^{5}$. Toutefois, la moitié des articles du présent numéro examinent des séries produites au $\mathrm{XX}^{\mathrm{e}}$ siècle en montrant que leur rapport à l'Histoire, à la mémoire et aux questions de représentation du phénomène guerrier est lui aussi riche, et souvent plus complexe qu'attendu.

\section{Violence, événement, héroïsme}

4 En outre, en tant que représentations audio-visuelles de la guerre, les séries télévisées se confrontent à la question de la mise en scène de la violence, ainsi qu'à la question de l'héroïsation, de la diabolisation ou de la dénonciation des soldats, des officiers, des ennemis, voire du phénomène guerrier en tant que tel. Dans un article consacré à la représentation de la guerre dans le cinéma de science-fiction nord-américain, Thierry Cormier rappelle que :

En tant que machine d'ombres et de lumières, le cinéma entretient presque ontologiquement avec le monde un rapport équivoque : «L'histoire disparaît à force d'être réécrite ", comme l'écrit Francis Vanoye, à propos de John Ford. Et pourtant, le film est aussi un document historique, en tant que photographie du contexte qui l'a vu naître.

(...) S'agissant de la guerre en tant qu'événement historique, toute représentation est questionnée sous l'angle du parti pris : « être pour ou être contre ». Il y a aussi des questions de l'ordre d'une possible spectacularisation qui se posent aux représentations de la guerre : trop voir la guerre c'est risquer la banalisation ou la fascination, mais ne pas assez la voir c'est risquer la déréalisation; ne montrer que les drames c'est oublier la quotidienneté, mais ne pas les montrer c'est nier la tragédie. Se pose également la question de la valeur de ces images, de leur rôle et de leur fonction vis-à-vis de l'événement représenté : entre information, spectacle et dénonciation. Le sujet " guerre » a ainsi atteint une dimension beaucoup plus symbolique en tant qu'il est devenu un moyen pour l'artiste d'évoquer plutôt que de raconter. (...) Enfin, face à la guerre, l'image, qu'elle émane de la télévision ou du cinéma, ne peut plus aujourd'hui se départir de son histoire ; tant pour le spectateur que pour le producteur, l'étendue des représentations guerrières suppose une réflexivité, consciente ou inconsciente, toujours plus prégnante ${ }^{6}$.

5 Si la guerre et le cinéma entretiennent une relation longue et complexe ${ }^{7}$, et que Thierry Cormier soulève des questions essentielles dès lors que l'on s'intéresse aux représentations visuelles de la guerre, on ne saurait appliquer exactement les mêmes cadres d'analyse aux séries télévisées. Par leur forme narrative longue et par le pouvoir des images qu'elles diffusent à un large public, redoublé par la force de la répétition ${ }^{8}$, les séries télévisées n'ont pas le même rapport à l'événement. Fictions de l'intime, avec des moyens techniques et financiers généralement inférieurs à ceux du cinéma, elles sont forcément du côté du banal et du quotidien. Leur récit d'une guerre s'inscrit forcément dans la durée, et par conséquent dans la nuance et les ambiguïtés. En cela, l'écriture sérielle se rapproche de l'écriture historienne, et s'éloigne de l'héroïsme du cinéma hollywoodien. 
6 La guerre est ainsi représentée au-delà, voire en dehors, de son expression paroxystique qu'est la bataille. Souvent par force (manque de moyens, censure) mais aussi par choix (grand public, parti pris artistique), les séries télévisées contribuent à mettre la violence de la bataille à distance, par l'ellipse, par le rire, ou bien par des « détours" tels que les expériences des civils, les aspects stratégiques, politiques ou diplomatiques, les temps morts des combats, ou encore la sortie de guerre ${ }^{9}$. Loin d'éloigner de la réalité du phénomène guerrier, ces détours complexifient au contraire notre compréhension, ou du moins notre appréhension, d'une expérience singulière.

\section{Guide de lecture de ce numéro}

7 Pour favoriser les liens et correspondances entre les analyses diverses et complémentaires contenues dans ce numéro, nous avons choisi de les regrouper en trois sous-ensembles. Les deux premières sous-parties examinent le rapport des séries télévisées à la mémoire, avec une première sous-partie qui interroge le rapport entre (re)construction(s) des imaginaires nationaux hors États-Unis, et une deuxième souspartie centrée sur la réécriture du passé récent dans les séries américaines, marquées par la guerre froide, le traumatisme vietnamien et ses résurgences lors des interventions au Moyen-Orient. La troisième sous-partie rassemble quant à elle des études sur la façon dont certaines séries ont, par choix ou par nécessité, mis la guerre à distance, nous permettant de mieux la regarder.

\section{Mémoires des guerres, guerres des mémoires}

8 Ainsi, l'article d'Olivia Carpi montre comment, à travers l'exemple du Chevalier de Pardaillan (Antenne 2, 1988), les séries télévisées du XX ${ }^{\mathrm{e}}$ siècle participent de la diffusion et du renforcement de stéréotypes et de lieux communs anciens concernant les grands épisodes de l'Histoire. Pourtant, dans cette adaptation des romans de cape et d'épée de Michel Zévaco, « dont l'objet n'est pas tant la restitution fidèle du passé que la création d'un monde culturellement exotique et aussi crédible que possible", l'historienne trouve « une lecture qu'on pourrait qualifier de 'politiquement correcte' des guerres de Religion dans la France des années 1980." En effet, la télévision française à cette époque devient républicaine plutôt qu'éducative, participant à un « changement de construction de l'identité nationale, moins fondée sur le rapport au passé que sur le partage de valeurs communes ${ }^{10}$ ». Cette "spaderie» est aussi emblématique de l'état de l'audiovisuel public après la privatisation de TF1, où les budgets des séries historiques ont été divisés par vingt. S'inscrivant dans la lignée initiée par Marc Ferro dans Cinéma et Histoire ${ }^{11}$ et prolongée par Isabelle Veyrat-Masson à propos de la télévision française ${ }^{12}$, Olivia Carpi conclut que

cette série télévisée peut être considérée comme le vecteur d'une mémoire collective, pas au sens historien du terme, c'est-à-dire objective, érudite, si possible véridique, mais au sens anthropologique, c'est-à-dire subjective, affective, parfois fantaisiste certes, et pourtant significative et rassembleuse ${ }^{13}$.

9 L'historien Jean-Marc Lafon s'intéresse lui aussi à une série des années 1980, Los Desastres de la Guerra (TV3, 1983), co-production franco-espagnole qui interroge la guerre d'Indépendance du début $\mathrm{du} \mathrm{XIX}^{\mathrm{e}}$ siècle à la lumière de la transition 
démocratique post-franquiste. A la veille de l'entrée de l'Espagne dans la CEE, la plume de Jorge Semprun et la caméra de Mario Camus amorcent une vision plus nuancée de la légendaire unité espagnole face à l'envahisseur français, en rappelant les espoirs de libéralisation et de modernisation suscités par l'arrivée des Bonaparte.

La série traduit assez fidèlement l'état d'esprit majoritaire de l'opinion espagnole du début de la démocratie: pacifisme consensuel, identité nationale ouverte, et sentiment rétroactif d'une victoire à la Pyrrhus en 1814 , signant la naissance des deux Espagne, l'une noire et l'autre rouge ${ }^{14}$.

On peut voir dans la guerre civile de 1936 l'aboutissement tragique de ce clivage. C'est ce conflit que revisite la série catalane Temps de silenci (TV3, 2001-2002), en insistant, nous explique Jennifer Houdiard, sur la dimension coloniale du conflit, et les conséquences de la défaite républicaine pour la langue et la culture catalane :

Temps de silenci raconte l'histoire de la Catalogne contemporaine comme celle d'une (re)construction après ce que l'on pourrait considérer comme un traumatisme originel : trois années de guerre, suivies de quatre décennies de répression visant à détruire la culture et l'identité catalanes, mais qui furent aussi quarante ans d'une résistance fertile et créatrice ${ }^{15}$.

Les deux hispanistes nous montrent ainsi comment la télévision espagnole post-Franco interroge la mémoire nationale et remet en cause la vision historique officielle du temps de la dictature.

L'angliciste Maggy Hary examine ensuite comment la mini-série de Peter Kosminsky The Promise (Channel Four, 2011) exhume le rôle oublié de la Grande-Bretagne dans les origines du conflit israélo-palestinien entre 1945 et 1948, replaçant la naissance de l'État d'Israël dans le contexte plus vaste de la décolonisation. Avec un récit qui effectue des allers-retours dans la chronologie, le réalisateur assume la dimension mémorielle de son propos, ainsi que l'ambiguïté politique et morale dans le fait de renvoyer dos à dos les terrorismes sioniste et palestinien :

Utilisant le son des sirènes comme effet de transition, Kosminsky fait se télescoper le passé et le présent et semble mettre au même niveau les terroristes des deux camps. De façon très polémique, ce passage de 1946 à 2005 semble sous-entendre que si l'insurrection sioniste contre l'occupant britannique put être perçue comme le premier acte d'une guerre d'indépendance, il serait désormais légitime d'envisager les attentatssuicides palestiniens comme des actes de résistance également justifiés. Cette séquence montre bien comment la mise en parallèle du passé et du présent sur laquelle la série repose fait constamment affleurer la concurrence de légitimité entre sionistes et Palestiniens qui utilisent en fait les mêmes méthodes à soixante ans d'intervalle ${ }^{16}$.

Les polémiques qui ont accompagné la diffusion de la série rappellent à quel point la mémoire de toutes les étapes de ce conflit encore en cours reste problématique.

\section{Le travail de mémoire dans les séries américaines, entre traumatisme et légende}

11 Les cinq articles de cette sous-partie s'intéressent aux séries américaines, qui s'inscrivent dans la tradition hollywoodienne résumée ainsi par Thierry Cormier : 
Les représentations cinématographiques de l'histoire aux États-Unis se déterminent selon deux axes complémentaires: d'une part, une approche généralement non didactique de l'événement historique, autrement dit affranchie de la mission d'enseignement; et d'autre part, l'usage d'un cadre avéré réel pour se concentrer sur les matrices idéologiques de la nation. Il n'est donc pas question de faits mais de valeurs, de l'essence même de l'idée américaine, l'Histoire devenant avant tout un cadre, voire un décor à des récits individuels capables de cristalliser des valeurs érigées en doctrines nationales. La reconstitution historique cède le pas à la construction ou à la déconstruction mythologique de l'Amérique, en évoquant, notamment, à l'intérieur d'un cinéma de genre, les fondements moraux qui ont présidé à la naissance et à l'évolution de la nation. Que ce soit le Western, le film de guerre ou le film de gangsters, les œuvres qui s'inscrivent dans ces registres participent de la tendance hollywoodienne à substituer aux yeux du public la légende à l'Histoire, l'imaginaire au réel ${ }^{17}$.

Néanmoins ces articles mettent en lumière l'évolution de cette dimension mémorielle et légendaire de la mise en récit de l'histoire américaine vers un discours de plus en plus nuancé et problématisé à mesure que l'on avance dans les années 1990 et 2000.

12 Ainsi, Julie Richard et Georges Caron comparent la représentation de la guerre froide dans des séries datant de la fin de ce conflit, Les Deux Font la Paire (Scarecrow and Mrs King) et MacGyver, avec celle qui en est faite dans The Americans, diffusée sur FX depuis 2013. Les deux historiens montrent ainsi que

la distance temporelle joue un rôle important dans la représentation du conflit. En effet, la représentation la plus dure, la plus noire et la plus violente se trouve dans la série la plus récente. Le temps écoulé donne donc plus de latitude aux intrigues. Les périls propres à cette guerre, ainsi que ses enjeux politiques, moraux et idéologiques, une fois éloignés, peuvent être abordés plus frontalement ${ }^{18}$.

De plus, chaque série témoigne d'une vision chronologiquement datée de l'identité américaine : la confiance retrouvée des années Reagan pour les deux premières, et les doutes propres à l'Amérique post-11 septembre pour la plus récente, diffusée sur fond de retour de l'antagonisme américano-russe.

13 La guerre froide sert également de toile de fond à la série de science-fiction du début des années 1990 Code Quantum, étudiée par Claire Cornillon, docteure en littérature comparée, dans l'article suivant. Toutefois, c'est la guerre du Vietnam et sa mémoire qui est au centre de cette relecture empathique et assez pacifiste d'une histoire récente des États-Unis (1953-1985). La spécialiste des séries semi-feuilletonnantes des années 1990 nous explique comment cette série s'inscrit parfaitement dans la tradition hollywoodienne du film d'Histoire décrite par Thierry Cormier et citée plus haut :

L'histoire dans Quantum Leap peut être à la fois iconique et individualisée. On ne touche jamais à la grande Histoire, on s'attache à la petite mais, en même temps, la Grande Histoire est toujours là abordée à travers un imaginaire culturel qui engage le téléspectateur ${ }^{19}$.

Cette réflexion sur la place de la mémoire est complétée par l'analyse d'une autre série de science-fiction des années 1990, Babylon 5, qui raconte cette fois des voyages dans l'espace plutôt que dans le temps, par Florent Favard, docteur en études cinématographiques et audiovisuelles. Tous deux montrent en effet qu'au sortir de la guerre froide, les États-Unis se livrent, à travers les séries télévisées (entre autres 
modes d'expression), à un examen de leur histoire récente, sans toutefois aller jusqu'à une autocritique poussée :

[Babylon 5] semble (...) piégée par ses ambitions : cette glorification du héros, cette vision individualiste de l'Histoire, couplée à son absence de questionnement sur l'hégémonie du capitalisme et de la démocratie libérale au sein de toute la galaxie, et ses références principalement anglo-saxonnes, en font un objet situé dans un contexte de production déterminé. De même qu'au cinéma, autre industrie culturelle hollywoodienne, Babylon 5 , série de science-fiction, est «par excellence le lieu de la critique sociale et politique » mais dans le même temps, cette critique n'est «tolérée que pour autant qu'elle entre dans ce que [la] société et ses membres peuvent supporter ${ }^{20}$.»

Toujours dans le domaine de la science-fiction, Frédéric Gai, doctorant en littérature, étudie comment la dimension eschatologique de The X-Files $(1993-2002$; 2016) se nourrit des guerres réelles menées par les États-Unis depuis 1945, et des traces douloureuses qu'elles ont laissées. La série se place ainsi, à ce titre et à bien d'autres, comme une série-charnière entre l'Amérique des années 1990 et l'Amérique post-11 septembre :

The X-Files est finalement symptomatique d'une époque et devient, en ce sens, charnière dans l'histoire des séries télévisées. Nous sommes en effet bien loin du "moralisme reaganien " des séries des années 80 , la série se situant du côté de la complexité et de la modernité artistique. Déjà torturés, ses héros ne sont à l'inverse pas encore les personnages plus sombres d' Homeland (Showtime, 2011-) ou de 24 (Fox, 2001-2014). Ils sont à la recherche d'un rapport phénoménologique à la vérité et sont encore très éloignés de l'anti-héros présent à partir du milieu des années 2000 dans des séries telles que Dexter (Sowtime, 2006-2013) ou encore Breaking Bad (AMC, 2008-2013). S'appuyant sur un couple héroïque porté par des valeurs, le récit construit une théorie d'une vérité qui se cache dans les êtres. La série devient alors symptomatique d'une idéologie qui vient agir comme une «consolation à la perte définitive de la transparence dans nos sociétés démocratiques ». Mais les créateurs de The X-Files ne pensent pas que cette recherche est une quête vaine. C'est un combat lui aussi perpétuel qu'ils mènent, un combat au cours duquel la série joue le rôle de garde-fou intellectuel et moral, notamment dans les périodes de dérives sécuritaires ${ }^{21}$.

La modification du rapport à la guerre des États-Unis entre l'avant et l'après-11 septembre est également sensible dans la transformation des codes hollywoodiens du film de guerre qui sont au cœur des deux mini-séries à gros budget produites par Steven Spielberg et Tom Hanks pour HBO, Band of Brothers (2001) et The Pacific (2010). Si toutes deux sont consacrées à la Seconde Guerre mondiale (dans son volet européen pour la première, et Pacifique pour la seconde), l'historien Guillaume Piketty insiste sur les différences entre ces deux opus sortis à près de dix ans d'intervalle, dont les différences «tiennent à la nature des sources employées, au type de combat livré, aux influences historiographiques et cinématographiques subies, ainsi qu'aux évolutions des contextes politique américain et international lors de la première décennie du XXI siècle. » Il montre notamment comment la représentation de la Seconde Guerre mondiale est influencée par les conflits postérieurs, rejoignant les analyses fondatrices de Marc Ferro ${ }^{22}$ :

Ainsi, la représentation du phénomène guerrier proposée par The Pacific est nettement plus complète que celle offerte par Band of Brothers. (...) Bien que tournée à la toute fin $\mathrm{du} \mathrm{XX}^{\mathrm{e}}$ siècle, Band of Brothers est ainsi la lointaine 
héritière de certaines superproductions des années 1960 et 1970 . Avec, sousjacente, l'idée de "Greatest Generation ", i.e. la génération de la Grande Dépression qui a tout surmonté et a fini par remporter la dernière guerre «juste » de l'histoire des États-Unis, le second conflit mondial. En refusant de considérer comme négligeables des éléments tels que, par exemple, l'environnement, les émotions et les traumatismes psychiques, les relations entre soldats et civils, ou encore le processus de sortie de guerre, The Pacific s'inscrit, elle, résolument dans l'après-Vietnam et ses syndromes de stress post-traumatique, l'après-Irak et l'après-Afghanistan ${ }^{23}$.

\section{Mettre la guerre à distance} principalement à la façon dont les séries télévisées mettent la guerre, en tout cas dans sa dimension d'affrontement militaire, à distance, par nécessité économique et/ou par choix artistique délibéré. Les deux premiers articles se penchent sur les comédies et la fonction à la fois cathartique et de mise à distance de la guerre par le rire et la dérision.

Ainsi, l'américaniste Dennis Tredy nous explique comment les comédies militaires américaines diffusées entre les années 1950 et 1970 ont fait office, derrière leur aspect léger et sans conséquence, de véritables "Chevaux de Troie culturels et politiques", interrogeant avec plus ou moins de force l'identité des États-Unis comme nation guerrière. Il nous montre en particulier comment le personnage du Sergent Bilko, créé dans les années 1950, a servi de modèles à de très nombreux récits mettant aux prises des sous-officiers (ou des médecins militaires comme dans $M^{*} A^{*} S^{*} H$ ) malins et roublards avec une hiérarchie bornée. Ces séries, diffusées sur les chaînes hertziennes alors hégémoniques, ont toutefois suivi les évolutions de l'opinion publique, offrant successivement une échappatoire bienvenue au conformisme des années 1950, puis une simple évasion optimiste lors des heures sombres du conflit vietnamien, pour enfin aider à sa digestion et à la compréhension du bouleversement socio-culturel des Sixties dans les années 1970 :

The Bilko model not only outlived the other forms of anti-establishment, anti-authority humor of the 1950s, but it continued after the program's cancellation, through afternoon syndication, primetime cartoons and especially a score of water-down avatars throughout the 1960s. For this second stage, the Bilko model was tweaked so as to strip it of its antiestablishment veneer and often reduced to slapstick escapism, creating quite the paradox-that of having no real treatment of the U.S. military at a time when U.S. forces were involved in a controversial war in Vietnam. (...) Though many might consider such escapism a much-needed palliative for a country at war, it seems safe to say that it was far too sugar-coated a pill to have any real effect on the patient's symptoms. (...) The palliative did come [in the 1970s:] $M^{*} A^{*} S^{*} H$ would thus prove that a true hybrid of wartime realism and irreverent slapstick comedy was not only possible, it was just what the doctor ordered ${ }^{24}$.

Justine Breton, docteure en littérature médiévale et spécialiste des représentations audiovisuelles de la légende arthurienne, nous explique ensuite comment Alexandre Astier, créateur-interprète de la série française Kaamelott (M6, 2005-2009), a su faire du manque de moyens pour représenter les combats une force, développant un discours aussi nuancé qu'accessible à un public familial :

TV/Series, 10 | 2016 
Le conflit et ses conséquences peuvent ainsi être représentés de façon comique, sans déshumanisation de l'ennemi ni des troupes, mais au contraire en valorisant toujours plus l'humain: les hommes sont capables d'erreur, d'incompréhension et de bêtise, et l'activité guerrière n'échappe pas à cette idée. Kaamelott porte donc un regard à la fois amusé et critique sur un passé mythique et réinventé, ainsi que sur la question plus globale de la guerre et des conflits armés ${ }^{25}$.

18 La question de l'ellipse est ensuite interrogée par la latiniste Julie Gallego. Elle nous montre comment la série Rome (HBO, 2005-2007) choisit d'employer son budget pharaonique non pas pour représenter directement les batailles antiques qui structurent son récit, mais pour représenter les causes et les conséquences psychologiques et politiques des affrontements, modernisant ainsi le genre cinématographique du péplum :

On comprend que ce qui est au cœur de l'œuvre, ce sont plutôt l'escalade vers la guerre, ses préparatifs, les manœuvres politiques pour la provoquer ou l'éviter, le goût des dirigeants pour le pouvoir, la difficulté du soldat à construire et reconstruire sa vie hors de la légion, la douleur des parents qui perdent un proche au combat, quel que soit son camp. Des thèmes universels afin de toucher les téléspectateurs de la série et de leur donner l'impression de partager le sentiment d'une expérience commune avec cet ailleurs étrange qu'est l'Antiquité romaine ${ }^{26}$.

19 L'angliciste Jean Du Verger analyse pour sa part comment l'adaptation de la tétralogie de Ford Madox Ford Parade's end par Tom Stopppard et la réalisatrice Susanna White pour la $\mathrm{BBC}$ parvient à rendre compte de l'expérience traumatisante de la Grande Guerre. Si l'essentiel des combats reste présenté de façon elliptique, la mise en scène souligne les multiples dimensions de leurs conséquences :

L'utilisation par la réalisatrice, Susanna White, d'images kaléidoscopiques renvoie au Vorticisme qui présente une vision fragmentée d'un monde tout en annonçant sa fin. Cela lui permet de traiter de manière oblique et fragmentée des réalités de la guerre qui ont entraîné le monde et les hommes dans un maelström dont ils ne sortiront pas indemnes - sans oublier le rôle essentiel des personnages et des dialogues qui peuvent être envisagés comme les surfaces qui réfractent la trame narrative en une multiplicité de points de vue.

(...) Par ailleurs, l'importance des fenêtres vient rappeler implicitement aux spectateurs que l'écran s'interpose entre eux et la narration, et que l'expérience des tranchées et de la guerre ne peut être réellement partagée. Les spectateurs en sont réduits au simple rôle de témoins «au seuil de cet enfer ", témoins qui demeureront à jamais de l'autre côté du miroir ${ }^{27}$.

Enfin, les historiens Zoé Grumberg et Clément Collard montrent comment dans sa cinquième saison, la série française Un Village français pousse la mise à distance du conflit armé jusqu'à la réflexivité. En mettant en abyme sa propre démarche de représentation de l'Occupation avec des personnages de maquisards qui «mettent en scène " un défilé symbolique le 11 novembre 1943 (inspiré d'un événement réel survenu à Oyonnax et filmé par les résistants du maquis de l'Ain), la série interroge les relations complexes entre guerre, jeu, engagement et commémoration ${ }^{28}$, sans se départir de son ambition historiographique : 
$\mathrm{Du}$ point de vue de son ambition historiographique, elle suit progressivement la constitution d'un maquis archétypal, en se focalisant sur quelques trajectoires exemplaires, envisageant ainsi le faisceau de facteurs pouvant mener un individu à la Résistance. Par ailleurs, cette saison permet de proposer une réflexion plus esthétique, autour de la question de la représentation; celle-ci est menée tout au long des dix premiers épisodes à travers l'importance accordée à l'écran aux activités théâtrales qui rythment, avec les entraînements, la vie quotidienne du maquis d'Antoine $\mathrm{Au}$ cours de ces dix épisodes, le jeu est devenu de plus en plus politique, jusqu'à se mettre au service d'une cause supérieure. Sa puissance n'en est pas altérée, puisque le défilé dans les rues de Villeneuve est l'occasion pour les maquisards de faire montre de leurs talents de comédiens, mais surtout de permettre au théâtre d'incarner, le temps de la "représentation », un esprit résistant. Celui-ci est rendu palpable par la mobilisation de références au travers des textes, ceux des chansons entonnées ou celui qui est déposé au pied du monument aux morts, par la démonstration de force que constitue la marche de dizaines de combattants dans une ville normalement occupée par les Allemands, mais surtout par la rencontre avec un public, soit une population civile véritablement affectée par le spectacle qui se déroule devant elle. Soit qu'elle soutienne, soit qu'elle désapprouve, elle n'est en tout cas pas indifférente à la représentation de la Résistance offerte par le maquis d'Antoine, qui s'inscrit pleinement dans la dimension symbolique de la lutte contre l'occupant ${ }^{29}$.

21 Evidemment, ce numéro n'a pas la prétention de faire le tour de la question de la représentation de la guerre dans les séries télévisées, et beaucoup d'autres œuvres restent à étudier, de même que d'autres questions méritent d'être soulevées. Néanmoins, par la variété des séries étudiées et par la diversité des angles et des disciplines convoqués, ce numéro vise à montrer la richesse des questionnements suscités par la représentation de la guerre dans les fictions télévisuelles.

\section{BIBLIOGRAPHY}

BOUTET Marjolaine, L'identité américaine face à la guerre. Etude de l'évolution des récits des guerres étrangères dans les manuels d'Histoire des États-Unis à destination du secondaire (1898-1991), Thèse de doctorat soutenue le 23 novembre 2009, Ecole Doctorale de Sciences Po, 3 volumes.

CABANES Bruno et Guillaume PIKETTY (éds.), « Sorties de guerre au XX $\mathrm{n}^{\circ} 3$, novembre-décembre 2007, URL : http://www.histoire-politique.fr/index.php? numero=03\&rub=dossier\&item=22, consulté le 29 novembre 2016.

CORMIER Thierry, « Guerre des images et imaginaires de guerre dans le cinéma de science-fiction nord-américain », Cycnos, Volume $22 \mathrm{n}^{\circ} 1$, mis en ligne le 13 octobre 2006. URL : http:// revel.unice.fr/cycnos/index.html?id=517, consulté le 28 novembre 2016.

ECO Umberto, «Innovation et répétition, entre esthétique moderne et post-moderne », Réseaux, $n^{\circ}$ 68, vol. 12, 1994 (trad. Marie-Christine Gamberini), p. 9-26. URL : http://www.persee.fr/doc/ reso_0751-7971_1994_num_12_68_2617, consulté le 28 novembre 2016. 
FERRO Marc, Cinéma et Histoire, Paris, Gallimard, 1993 [1977].

HATCHUEL Sarah et Marjolaine BOUTET, « Henri VIII en série : Les Tudors et Wolf Hall », conférence au Musée du Luxembourg, 12 mai 2015.

RICœUR Paul, La mémoire, l'histoire, l'oubli, Paris, Seuil, 2000.

TOUBIANA Éric-Pierre, « La guerre est-elle un jeu ?», Topique, 1/2008 ( $\left.{ }^{\circ} 102\right)$, p. 163-180. URL :

http://www.cairn.info/revue-topique-2008-1-page-163.htm ; DOI : 10.3917/top.102.0163, consulté le 29 novembre 2016.

VEYRAT-MASSON Isabelle, Quand la télévision explore le temps, Paris, Fayard, 2000.

VEYRAT-MASSON Isabelle, Télévision et Histoire, la confusion des genres. Docudramas, docufictions et

fictions du réel, Bruxelles, INA-de Boeck, collection « Recherches », 2008.

VIRILIO Paul, Guerre et cinéma, Paris, Les Cahiers du Cinéma - Essais, 1991.

\section{NOTES}

1. Isabelle Veyrat-Masson, Quand la télévision explore le temps, Paris, Fayard, 2000, p. 9.

2. Cf. Marjolaine Boutet, L'identité américaine face à la guerre. Etude de l'évolution des récits des guerres étrangères dans les manuels d'Histoire des États-Unis à destination du secondaire (1898-1991), Thèse de doctorat soutenue le 23 novembre 2009, Ecole Doctorale de Sciences Po, 3 volumes.

3. Paul Ricœur, La mémoire, l'histoire, l'oubli, Paris, Seuil, 2000, p. 170

4. Ibid., p. 175-178 et p. 339-358.

5. Cf. Sarah Hatchuel et Marjolaine Boutet, «Henri VIII en série : Les Tudors et Wolf Hall», conférence au Musée du Luxembourg, 12 mai 2015.

6. Thierry Cormier, «Guerre des images et imaginaires de guerre dans le cinéma de sciencefiction nord-américain ", Cycnos, Volume $22 \mathrm{n}^{\circ} 1$, mis en ligne le 13 octobre 2006. URL : http:// revel.unice.fr/cycnos/index.html?id=517, consulté le 28 novembre 2016.

7. Voir notamment Paul Virilio, Guerre et cinéma, Paris, Les Cahiers du Cinéma - Essais, 1991.

8. Umberto Eco, «Innovation et répétition, entre esthétique moderne et post-moderne », Réseaux, $\mathrm{n}^{\circ}$ 68, vol.12, 1994 (trad. Marie-Christine Gamberini), p. 14. URL : http://www.persee.fr/doc/ reso_0751-7971_1994_num_12_68_2617, consulté le 28 novembre 2016.

9. Sur la richesse de ce dernier concept, mis au point par Bruno Cabanes, cf. notamment Bruno Cabanes et Guillaume Piketty (éds.), « Sorties de guerre au XXème siècle », Histoire@Politique, n 3, novembre-décembre 2007, URL: http://www.histoire-politique.fr/index.php? numero=03\&rub=dossier\&item=22, consulté le 29 novembre 2016.

10. Pascale Goetschel, "Compte-Rendu de Quand la télévision explore le temps... », Vingtième Siècle, vol. 72, n 1, 2001, p. 163, cité par Olivia Carpi, «L'art du stéréotype : la représentation des guerres de Religion dans la série Le Chevalier de Pardaillan de Josée Dayan (Antenne 2, 1988) », TV/ Series, $n^{\circ}$ 10, décembre 2016. URL : http://tvseries.revues.org/1921

11. Marc Ferro, Cinéma et Histoire, Paris, Gallimard, 1993 [1977].

12. Veyrat-Masson, op.cit. Voir aussi, du même auteur : Télévision et Histoire, la confusion des genres. Docudramas, docufictions et fictions du réel, Bruxelles, INA-de Boeck, collection « Recherches », 2008.

13. Carpi, art.cit.

14. Jean-Marc Lafon, «Hommage artistique ou tentative pionnière de révision historique ? La Guerre d'Indépendance espagnole (1808-1814) au prisme de la série Los Desastres de la Guerra (TV3, 1983) », TV/Series $n^{\circ} 10$, décembre 2016. URL : http://tvseries.revues.org/1752 
15. Jennifer Houdiard, "Entre traumatisme originel et choc fondateur: la Guerre Civile espagnole dans la série catalane Temps de silenci (TV3, 2001-2002) », TV/Series, $\mathrm{n}^{\circ} 10$, décembre 2016. URL : http://tvseries.revues.org/1858

16. Maggy Hary, "Terrorisme ou guerre de libération? Lecture critique du conflit israélopalestinien dans The Promise de Peter Kosminsky (Channel Four, 2011) », TV/Series [En ligne], 10| 2016, mis en ligne le 01 décembre 2016, consulté le 29 novembre 2016. URL: http:// tvseries.revues.org/1863

17. Cormier, art.cit.

18. Julie Richard et Georges Caron, «Guerre froide/ guerres froides : les représentations de l'opposition américano-soviétique dans Scarecrow and Mrs. King (CBS, 1983-1987), MacGyver (ABC, 1985-1992) et The Americans (FX, 2013-) ", TV/Series $n^{\circ} 10$, décembre 2016. URL: http:// tvseries.revues.org/1832

19. Claire Cornillon, "La représentation des guerres et de l'histoire américaine de la seconde moitié du XXe siècle dans Code Quantum (Quantum Leap, NBC, 1989-1993)», TV/Series, n 10, décembre 2016. URL : http://tvseries.revues.org/1937

20. Éric Dufour, Le Cinéma de science-fiction, Paris, Armand Colin, 2011, p. 189, cité par Florent Favard, « War Without End : le devoir de mémoire dans Babylon 5 (PTEN>TNT, 1993-1998) », TV/ Series, $\mathrm{n}^{\circ}$ 10, décembre 2016. URL : http://tvseries.revues.org/1855

21. Frédéric Gai, « Le fracas silencieux de la guerre dans The X-Files (1993-2002; 2016) », TV/Series, $\mathrm{n}^{\circ}$ 10, décembre 2016. URL : http://tvseries.revues.org/1887

22. Marc Ferro, «Le film, contre-analyse de la société ?» (1971), Cinéma et Histoire, Paris, Gallimard, 1993, p. 40.

23. Guillaume Piketty, "La Seconde Guerre mondiale dans Band of Brothers (HBO, 2001) et The Pacific (HBO, 2010) », TV/Series, $n^{\circ} 10$, décembre 2016. URL : http://tvseries.revues.org/1897

24. Dennis Tredy, “"'War... What Is It Good For ?' Laughter and Ratings” : Sgt. Bilko, $M^{*} A^{*} S^{*} H$ and the Heyday of U.S. Military Sitcoms (1955-75) », TV/Series, n 10, décembre 2016. URL : http:// tvseries.revues.org/1764

25. Justine Breton, « «Rire et faire rire de la guerre: Kaamelott (M6, 2005-2010) ou la mise à distance du conflit » ", TV/Series, $n^{\circ} 10$, décembre 2016. URL : http://tvseries.revues.org/1796

26. Julie Gallego, « Guerre des Gaules et guerres civiles dans la série Rome (HBO, 2005-2007) », TV/ Series, $n^{\circ} 10$, décembre 2016. URL : http://tvseries.revues.org/1873

27. Jean du Verger, «Parade's End (2012), ou le miroir fragmenté d'un monde en décomposition », TV/Series, $\mathrm{n}^{\circ}$ 10, décembre 2016. URL : http://tvseries.revues.org/1814

28. Sur ce sujet, lire notamment Éric-Pierre Toubiana, "La guerre est-elle un jeu? ", Topique, 1/2008 ( $\mathrm{n}^{\circ}$ 102), p. 163-180. URL : http://www.cairn.info/revue-topique-2008-1-page-163.htm ; DOI : 10.3917/top.102.0163, consulté le 29 novembre 2016.

29. Clément Collard et Zoé Grumberg, «Le maquis d'Antoine dans Un Village français : un théâtre de la Résistance ? », TV/Series, n 10, décembre 2016. URL : http://tvseries.revues.org/1844

\section{AUTHOR}

\section{MARJOLAINE BOUTET}

Marjolaine Boutet est maitre de conférences en Histoire contemporaine à l'université de Picardie-Jules Verne. Spécialiste de l'histoire des séries télévisées, elle est l'auteure de Les Séries 
Télé pour les Nuls (First, 2009) et Cold Case : la mélodie du passé (PUF, 2013) ainsi que co-auteure de Seriescopie : guide thématique des séries télévisées (Ellipses, 2011) avec Pierre Sérisier et Joël Bassaget. Elle s'intéresse particulièrement à la représentation de l'Histoire et des conflits dans les séries télévisées, qu'ils soient militaires, politiques ou symboliques.

Marjolaine Boutet is Associate Professor in Contemporary History at the University of PicardieJules Verne. She specializes in TV series and is the author of Les Séries Télé pour les Nuls (First, 2009) and Cold Case : la mélodie du passé (PUF, 2013), as well as a co-author of Seriescopie : guide thématique des séries télévisées (Ellipses, 2011) with Pierre Sérisier and Joël Bassaget. Her research focuses on the representation of History and conflicts on screen. 